\title{
Problems of Clinical Pharmacology in the United States
}

Edward A. Carr, Jr., M. D.*

It is an honor to have the opportunity to contribute to the Japanese Journal of Clinical Pharmacology. Let me begin by wishing the Journal and its sponsoring Society a very long and useful career.

In 1953, as the result of a decision by Professor M. H. Seevers, a clinical pharmacology program was initiated at the University of Michigan. The development of clinical pharmacology up to the mid-point of the 20th Century will first be reviewed briefly here, and the subsequent developments during the last 20 years will then be discussed. Problems of clinical pharmacology can be described most clearly by a review of its development.

However, before discussing these developments, it is important to consider the significance of American experience for the very important new development of clinical pharmacology in Japan. Increased knowledge of clinical pharmacology is necessary to safeguard the public health, and clinical pharmacologists can make important contributions in medical schools, hospitals, industry and government. Therefore, clinical pharmacology programs must be concerned not only with research and clinical service, but also with the training of many additional individuals in this field. The education of practicing physicians in more rational use of drugs is also essential. Certain problems will inevitably arise whenever a clinical phar-

\footnotetext{
* From the Departments of Pharmacology and Internal Medicine, and the Upjohn Center for Clinical Pharmacology, University of Michigan, Ann Arbor, Michigan, U.S.A.
}

macology program develops in any country, e. g., the relation between clinical pharmacology and basic pharmacology. Ideally, a university pharmacology department should be a " center for knowledge of how drugs work" (1) and clinical pharmacology should be based, at least partly, in a pharmacology department. Nevertheless, whenever any problem requiring solution arises in two different countries, the solutions will not be the same, even when both countries are, like Japan and the United States, scientifically advanced. Differences in national culture will affect the methods of solving the problems. As an example, the willingness of patients to serve as research subjects may differ from country to country. Even though the controlled clinical investigation of drugs (the modern clinical trial) is actually far safer than the unscientific methods used in the past, patients expect more benefit from drugs now and are less willing to accept adverse reactions to them. This is a significant problem, as improved clinical study of new drugs is an important area in clinical pharmacology.

But certain differences can be the result of conscious decisions. For example, if universities play a larger role in establishment of clinical pharmacology in one country, while another country utilizes its pharmaceutical industry as the major base for clinical pharmacology, differences in result will not represent differences in national culture but rather differences between universities and industry. The Japanese Society for Clinical Pharmacology will determine which choices 
in Japan should be similar to those made in the United States and which choices should be made differently. Thus, the decision to combine clinical research with basic pharmacologic research in clinical pharmacology programs was, I believe, a wise decision. It is recommended that clinical pharmacology programs always provide laboratory facilities for basic research in pharmacology and simultaneous clinical opportunities for its staff and students. On the other hand, the attempt to develop extensive teaching of clinical pharmacology for medical students, in addition to training and research for advanced individuals, has thus far produced insufficient results in the United States (2). Practical arrangements for increasing this teaching still need to be improved.

Although Dr. James Lind investigated, in a controlled clinical trial, the effect of citrus fruit in scurvy during the 18th Century in England, 200 years elapsed between his work and the development of a clinical pharmacology program by Gold and Modell in the United States. At least five reasons caused this delay: lack of sufficient knowledge of the causes of disease to provide a basis for rational therapeutics; lack of sufficient chemical and physiological background for development of pharmacology; problems in the development of pharmacology; rise of therapeutic nihilism; effect of outmoded academic tradition.

The first and second of these causes are well-known and will not be discussed further here. But the irregular history of the development of pharmacology deserves brief comment. By the beginning of the 20th Century Ehrlich had established rational pharmacology in the chemotherapy of infection, and his work had prompt clinical importance. But pharmacology entered a sluggish period after this, for additional chemotherapy, extending into bacterial diseases, was slow to develop. The isolation of various hormones proved important in therapeutics, but such hormones were initially confined to use in specific deficiency states. Sulfonamides probably contributed more to the development of pharmacology, both basic and clinical, than any other class of compound introduced between 1925 and 1950. They demonstrated again the effectiveness of Ehrlich's plan for rational drug development, proved the importance of drug metabolism, showed that certain results could be transferred from a model animal system to human medicine, and also renewed interest in certain problems of pharmacology that could not easily be solved in animals. Thus, the observation that patients receiving sulfonamides sometimes develop allergic manifestations similar to those seen after use of antiserum provided a new stimulus to studies in allergy to drugs. Subsequently, chemotherapy was extended by development of antibiotics, corticosteroid drugs, in large doses, were found effective in the treatment of many disorders that were not caused by adrenal insufficiency, and a cascade of new drugs began to pour down on medicine in the late 1940s. The increasing importance of pharmacology, with its emphasis on selective toxicity, became clear to some individuals. The important point is this: when basic pharmacology is weak, clinical pharmacology does not advance well. And attempts to make advances in basic pharmacology, without paying proper attention to clinical pharmacology, result in a very deformed basic pharmacology. Balanced attention to each permits a rapid expansion of each. When Krayer and others were using the heart-lung technique in animals as an important tool in pharmacology, Gold and Modell were developing their classic studies of human pharmacology of cardiac glycosides. Beecher and Lasagna investigated newer analgesics in man in the $1940 \mathrm{~s}$, and this was the decade that witnessed the expansion of significant (i. e. primate) studies of addiction to narcotics in the laboratory at Michigan. By 1953 advances in basic pharmacology and clinical needs had made the establishment of clinical pharmacology programs not only possible but essential.

Nevertheless, clinical pharmacology was still more of a promise than a reality when 
we began the program at Michigan in 1953. To my knowledge, only two regular programs existed at that time - the program of Gold and Modell at Cornell and the program of Lasagna at Johns Hopkins University. When Dr. Seevers and I discussed the next steps to be taken during the summer of 1953, the problems of therapeutic nihilism and outmoded academic tradition still faced us. Therapeutic nihilism had its basis, to a considerable extent, in the serious adverse effects that had often occurred in the past as a result of ill-advised attempts at therapy with various agents which had not received proper pharmacologic study. The introduction of some drugs had lead to disastrous consequences that prejudiced physicians against all drugs(3). But by 1953, advances in basic pharmacology offered considerable encouragement.

Academic tradition sometimes delayed development of clinical pharmacology, for the proper study of clinical investigators for many years was considered to be the mechanism of disease and its various diagnostic aspects. No one can deny the importance of such studies, but exclusive interest in these areas led to neglect of interest in human pharmacology. The introduction of a clinical pharmacology program at the University of Michigan in 1953 was largely a result of the fact that the Chairman of the Department at Michigan was one of the few individuals in the country who thought that clinical pharmacology had any future at that time. Developments in pharmacology and clinical medicine had made such programs essential, but the failure of many universities to change had made such programs practically impossible in those universities.

Although I have obvious personal interest in subsequent developments at Michigan, many American universities now have programs in clinical pharmacology. After briefly describing the developments at Michigan from 1953 to 1970 , it will be important to consider the much larger subject of developments throughout the United States during that period.

Before coming to Michigan, I had obtained some experience in teaching human pharmacology to medical students at Harvard, encouraged by Drs. Krayer, Castle, and Riggs. Dr. Seevers strongly encouraged continuation of this teaching at Michigan, and provided opportunity for additional teaching of medical students. Such courses were well received, but several major changes in the entire curriculum of the medical school at Michigan took place in subsequent years. With each abrupt change in the curriculum for the whole school, therapeutic nihilism and academic tradition required revision of all our plans. At present, a monthly conference in human pharmacology is held at one teaching hospital for medical students at Michigan, an elective course in clinical and basic pharmacology is available to medical students during their last clinical year, and a human pharmacology seminar is given to advanced students in clinical pharmacology. We have recently given various evening lectures for interns and residents.

Since 1959, a Training Program in Clinical Pharmacology at Michigan has provided advanced instruction, including research experience, to individuals who have already had clinical training and wish to increase their knowledge of pharmacology. This program, supported by the National Heart Institute, provides stipends for American trainees (advanced students) and various other funds, including Merck Fellowships, a World Health Organization Fellowship, etc. have at times provided support for similar trainees from other countries. It is extremely important that firm arrangements be made in advance to provide an academic position in his own country to every qualified individual who completes a special training program in clinical pharmacology. If the individual returns to his own country, after receiving advanced training, and does not find such a position, he loses the enthusiasm and dedication which he was prepared to bring to the new program in his own country. The failure to create a large number of young clinical pharmacologists to start additional programs in the United States has also been 
a problem here. Individuals who have received training in clinical pharmacology often return to entirely clinical work or entirely basic pharmacology, without sufficiently using their training to combine the two areas. The relation between the development of advanced training programs to produce more clinical pharmacologists and the progress of medicine in general is only beginning to appear, but may become much more obvious in the future (4).

Clinical pharmacologists can perform a very great service by improving the methods by which new drugs are evaluated in man. One of the major changes needed in medicine is the replacement of unscientific methods by well designed clinical trials. The design of clinical trials involves ethics, strategy and tactics (5). Ethical considerations include careful attention to informed consent by the patient, especially when the work is truly experimental, involves any significant risk, or does not provide direct benefit to the patient himself. Strategic considerations include: consultation with a statistician in advance of the trial, whenever possible, in order to choose the simplest experminental design that is appropriate for the clinical trial (this includes such questions as random sampling, "double blind" experiments, placebo or other control, etc); the decision whether to compare two drugs at doses that will give equal therapeutic effect, or doses that are expected to give equal adverse effects; attention to the result of previous animal studies, which may provide at least a clue to the outcome in man, etc. Tactical considerations include: preparation for possible adverse effects, including emergency measures; calculation of the number of patients that will be required to give a significant result; consideration of the possibility of a simultaneous cooperative study in several clinics; the problem of diagnosis and exclusions (e.g. what diagnostic requirements are necessary before a patient can be accepted as having the disease which is under study, and what characteristics might exclude a patient from the study); possible ways of getting quantitative or semi-quantitative data from careful clinical observation, as well as laboratory findings; establishment of a system of grading the severity of disease and the degree of improvement, etc.

If clinical pharmacology in Japan, or in any country, provides more scientific, reliable, and safe clinical trials of drugs, this will represent a major achievement.

Limitation of space for research clinics and laboratories had become a severe problem in the Michigan program by 1963 . Such a problem has also been very troublesome in many other programs (6). In response to a request from the president of the University, Dr. E. Gifford Upjohn announced in 1966 a gift of money to the University, to permit construction of a building devoted entirely to clinical pharmacology. This building, the Upjohn Center for Clinical Pharmacology, was dedicated in 1970. It is owned entirely by the University and completely administered by the faculty of the Medical School. The Center permits the study of new and old drugs, diagnostic, prophylactic and therapeutic agents, stable and radioactive drugs, early phase and late phase clinical trials in humans, and studies of drugs made by any manufacturer. The Center is supported by the University and the National Institute of General Medical Sciences. The Center provides office and laboratory space for the several investigators who form its staff; each investigator has his own departmental appointment in addition to his appointment to the staff of the Center. The Departments of Pharmacology, Internal Medicine, Biochemistry, Pediatrics and Anesthesiology are currently represented. The Center also provides space for individuals receiving training in clinical pharmacology. A third function of the Center is to provide a clinical area in which any qualified member of the faculty, upon vote of the Operating Committee of the Center, may conduct his own studies in human pharmacology. The Center is for ambulatory patients, but bed patients may be brought to the Center during the day from the adjacent University Hospital. 
Also, patients requiring bed care can be admitted to the Clinical Research Unit of the Hospital, which is located very near the Clinical Pharmacology Center.

At present, the Staff of the Center are studying the development of better methods for analysis of the concentration of analgesic agents, cardiac glycosides, and lidocaine in human plasma; metabolism of anesthetic agents in man; effect of drugs on connective tissue diseases; myocardial uptake of radioactive compounds in animals and man for diagnostic use in various conditions, including the rejection reaction of heart transplants; effect of drugs on synthesis of heme; studies of retinal metabolism; and the distribution and effect of corticosteroid drugs in children, patients with renal disease, and other special states. Other research projects conducted by the medical faculty in the Clinical Pharmacology Center include the effect of various drugs upon the release of insulin, growth hormone, and glucagon; evaluation of drugs in treatment of hypertension; differences in rate of absorption of different formulations of the same drug (e.g. Warfarin); use of drugs in Parkinsonism, etc.

Each staff member conducts teaching and provides clinical services in his own department. The number of accidental and suicidal poisoning has increased in Michigan, as in other areas of the world; the problem of drug abuse has increased throughout the world (7); adverse reactions to drugs have become more frequent with increasing use of potent compounds; and problems of toxicology require increasing attention (8). Poison Control Centers have therefore been developed in many hospitals, as well as programs for reporting adverse reactions to drugs. In the last two years, these activities have been combined at Michigan in the form of a Poison-Adverse Reaction Team. Any poisoning or serious adverse reaction may be referred to this team. A physician member of the team is always on call to provide emergency consultation service to patients at the University Hospital. The physican who is on call may himself seek additional advice from various pharmacologists, psychiatrists, and others who are members of the team. The clinical pharmacology staff provides considerable clinical service on this team, in addition to their other clinical duties.

Approximately one third of the medical schools in the United States now have clinical pharmacology programs. In 1964 the National Institute of General Medical Sciences defined clinical pharmacology as the study of drugs in man, using the same scientific principles that are valid in laboratory pharmacology, with special consideration of the unique problems and opportunities of human studies. A clinical pharmacologist must also help to develop clinical pharmacology as a discipline in its own right. This requires careful consideration of the way he spends his time. In addition to devoting a portion of his time to the pharmacology department and another portion to clinical activities, he should have approximately one-third of his time available for interdisciplinary activities. The process of combining clinical medicine and pharmacology is in itself a time-consuming process (9). The many demands upon his time are obvious from the recent report of the World Health Organization (10), indicating the research, teaching and service that is needed in this area.

Among the events that led to the recent increase in the number of clinical pharmacology programs in the United States were: increase in number of available drugs; increase in dubious therapeutic claims; need for additional pharmacologic knowledge in support of new fields, e.g. organ transplantation; eventual recognition that the universities had failed to provide sufficient teaching of clinical pharmacology and had left the postgraduate education of physicians to the pharmaceutical industry, without university participation; eventual recognition of the need to decrease the gap between clinicians and basic investigators; increased support of university programs from industry and government; finally, as a most unfortunate stimulus, the thalidomide tragedy. In the 
United States, the founding of the Journal, "Clinical Pharmacology and Therapeutics" by Dr. Walter Modell represented an important advance. For physicians who had previously been exposed to articles that were " often difficult to evaluate...(and) not infrequently repudiated "(11) the Journal proved a very refreshing change. The establishment of a Journal of Clinical Pharmacology in Japan is a very encouraging development.

Progress in clinical pharmacology has been aided by availability of statistical methods suitable for study of small numbers of patients, computer methods for large studies, the "double blind" technique, radioactive tracers, and better methods for analysis of drugs in body fluids.

In spite of these stimuli, the development of clinical pharmacology in the United States remains incomplete. An important statement of its needs resulted from the Basin Harbor Conference in 1965 (6). The conference stressed the important role of medical school deans in promoting inter-departmental activities such as clinical pharmacology, the need for stable support of young investigators, the importance of adequate space, the budgeting of funds for clinical pharmacology. A through discussion between participating clinical departments and the pharmacology department should precede the organization of a clinical pharmacology program in any school.

Clinical pharmacology programs in the United States are still relatively small and usually depend upon a liaison between one major clinical department (often Internal Medicine but with increasing participation by Pediatrics, Anesthesiology, etc) and Pharmacology. Some programs stress one particular area of clinical pharmacology. Other programs, including the one at Michigan, permit a trainee to work in any one of a wide number of areas; if necessary, arrangements are made to allow him to conduct part of his training under a member of the faculty who is not a member of the clinical pharmacology staff, if that member can provide the most expert supervision. A third type of program, in a few schools, does not stress the importance of one particular clinical area for each individual in training. Since clinical pharmacologists must compete with other clinicians, and it is difficult to master the techniques of many medical specialties in one lifetime, there is increasing tendency to provide a broad background in pharmacology to all clinical pharmacologists, but encourage each individual to learn the details and techniques of one specific clinical area.

Clinical pharmacology develops best in an environment of constant teaching. The teaching of physicians who have already graduated from medical schools poses a serious problem, as a physician who wishes to learn how to treat heart disease, for example, is more likely to ask a cardiologist than a clinical pharmacologist. The established specialties have of course contributed excellent research in human pharmacology and this should continue to be encouraged. This is one of the reasons for the arrangement at Michigan, which permits various specialties to conduct clinical trials within the Clinical Pharmacology Center. But, as the total number of drugs increases, and the possibility of important drug interactions increases, while knowledge of drug metabolism and the ability to evaluate therapeutic claims become more important every day, physicians must improve their education in the area of human pharmacology or their patients will suffer.

In a few medical schools clinical pharmacology has not been inter-disciplinary. On a few occasions, it has been regarded as a theoretical laboratory subject, with a clinical pharmacologist serving only as a consultant to clinicians. In other cases, the opposite extreme has been reached, and clinical pharmacology programs have developed almost entirely within clinical areas, allowing little participation of pharmacology departments. Neither of these extremes has proved as successful as the intermediate approach that combines pharmacology with clinical medicine. The clinical parmacologist must continue his attempts to transfer the knowledge gained in the laboratory to the welfare of patients. Although this transfer is difficult, 
and accounts for many of the problems in recruiting young men to enter this field, the combination of pharmacology and clinical medicine is the essence of clinical pharmacology. Although the relative importance of service to patients and participation in research will differ from country to country and even from school to school, depending upon the needs of the people involved, one type of activity should not completely cancel out the other. Experience has also suggested that two other extremes be avoided: the clinical pharmacologist should not attempt the development of a huge program that combines too many aspects of medical education at one time, and clinical pharmacologists whose chief research is in the area of clinical trials should have the opportunity to improve the design and methods of clinical trials, and not be restricted to routine work.

Certain aspects of clinical pharmacology are probably universally important. These include the need to combine pharmacology and clinical medicine, the desirability of instituting a teaching program at once, the need to improve the education of physicians who have already graduated from medical school, the importance of providing stable support for young investgators, and the desirability of working with both government and industry in a program that is centered in a university.

I wish to thank the Japanese Journal of Clinical Pharmacology for the kind invitation to contribute this paper and wish you great success in your endeavors.

\section{REFERENCES}

1) Maren, T. H., Pharmacology: Its Nature in Medicine. Science, 161, 442-444 (1968).

2) Carr, E. A. Jr. : A Short Course in Clinical Pharmacolgy. Clinical Pharmacology and Therapeutics, 11, 455-459 (1970).

3) Modell, W.: Mass Drug Catastrophes and the Roles of Science and Technology. Science, 156, 346-351 (1967).

4) Carr, E. A. Jr.: Training in Clinical Pharmacology and Medical Progress: Is There a Dose-Response Relation?. Proceedings 3rd International Pharmacology Meeting (Sao Paulo, Brazil) ; Pergamon Press, New York (1968).

5) Carr, E. A. Jr.: Canadian Medical Association Journal, Practical Considerations in the Design of Clinical Trials. 98, 307313 (1968).

6) Lasagna, L., Clinical Pharmacology: Science, Present Status and Future Development. 152, 388-390 (1966).

7) Seevers, M. H. : Drug Dependence and Drug Abuse. A World Problem. The Pharmacologist, 12, 172-181 (1970).

8) Brodie, B. B., Cosmides, G. J. and Rall, D. P. : Toxicology and the Biomedical Sciences. Science, 148, 1547-1554 (1965).

9) Carr, E. A. Jr. : Proposed Aims, Organization and Activities of a Division of Clinical Pharmacology. Clinical Pharmacology and Therapeutics, 4, 587-595 (1963).

10) Clinical Pharmacology. Scope, Organization, Training. World Health Organization Technical Report Series No. 446, WHO, Geneva, 1970. (also published in Pharmacologia Clinica, 2, 241-248 (1970).

11) Modell, W.: Editorial. Clinical Pharma. cology and Therapeutics, 1, 1 (1960). 